\title{
Study on the Stability of High-Speed Turning Braking Based on the Hardware-in-the-Loop Test
}

\author{
Yi LU, Jie LU, Jia-wei WU, Bin GUO
}

\begin{abstract}
During the tire cornering braking process, busses easily cause traffic accidents such as slewed or tail flick if the tire is not locked. Therefore, in the process of cornering braking, the theoretical controlled slip rate of approximately 0.2 is not sufficient. To improve the stability of the vehicle, the Hardware-in-the-Loop Test is introduced. The fuzzy PID algorithm is used to calculate the tire slip rate of four tires using the actual yaw velocity and sideslip angle values and the expected difference among the values. The high-speed vehicle was tested on high- and low-adhesion roads. The study shows that this method outputs the new slip rate, improves the vehicle stability in the loss of braking efficiency, further perfects the study of the antilock braking system and has practical significance.
\end{abstract}

Keywords: hardware-in-the-loop; sideslip angle; stability; yaw velocity

\section{INTRODUCTION}

Recently, there have been several ABS studies on vehicle cornering braking conditions but notably few on the stability. However, the vehicle braking stability is the most significant index when the vehicle is moving [1]. The vehicle braking stability is used to limit the scope of the sideslip angle of the wheels and control the yawing moment to avoid losing control of the vehicle when the vehicle brakes or turns at a high speed. In addition, the stability makes the vehicle return to its original track through active intervention while emergent.

There are two methods to study the vehicle stability: pure simulation and vehicle experiment $[2,3]$. Cornering braking is a complicated process, which cannot be fully analyzed by the pure simulation method. Moreover, accidents may occur in a real-vehicle road experiment because of the road condition, controlling method and other factors. The hardware-in-the-loop method to study the ABS can avoid some defects of the pure simulation, e.g., low precision, lack of intuition and high risk. This joint simulation combines software and real vehicles to greatly improve the experiment authenticity $[4,5]$.

Normally, in the condition of low-speed turning, limiting the slip rate to 0.2 may satisfy the demand of vehicle stability controlling standard. However, when the vehicle is at high speed, a more effective method to adjust the vehicle slip rate using the yaw velocity and sideslip angle as the controlling index to correct the slip angle is necessary [6].

\section{EXPERIMENTAL}

\subsection{Hardware-in-the-Loop Test Structure and Operating Principle}

In the hardware-in-the-loop test, the actuator control and control algorithm should simultaneously work to ensure good real-time performance of the test. Comparing with other real-time development platforms, the xPC Target is more economically efficient and open [7]. The entire system includes an air brake system, the host machine and the target machine. The host machine uses MATLAB Simulink to write the vehicle dynamic model and the ABS controlling model and simulate; the target machine handles the code that the host machine produces. The two machines are connected by TCP/IP. Fig. 1 shows the Hardware-in-the-loop simulation test system schematic.

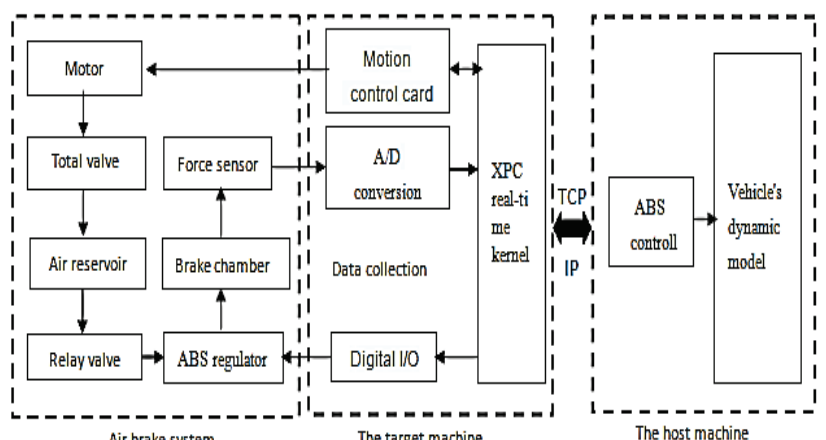

Figure 1 Hardware in the loop simulation test system schematic

The working principle of the system is shown in Fig. 2. After braking, the total valve (7) outputs air pressure after motor 20 is on; then, gas in the air reservoir $(4,5$, and $6)$ is quickly transported to the relay valve $(8,13$, and 15$)$ and enters the brake chamber, where the air pressure increases and produces a braking force output. After receiving the signal, the force sensor $(10,12,17,19$, and 22 ) begins the $\mathrm{A} / \mathrm{D}$ transformation through a data collector in the target machine. In the $\mathrm{XPC}$ target environment, the signal is transferred to the model in the target machine, and the vehicle dynamic model begins to calculate. Through the I/O part of the data-collecting card, the ABS controlling model provides a signal to the ABS regulator $(23,24,25$, and 26) according to the real-time calculation result. In the test, a high-speed solenoid valve drive chip uses TLE6228 and has two high-speed solenoid valves: intake valve and exhaust valve. The drive chip increases, maintains or decreases the pressure according to the open or close condition of the valves to prevent the wheels from locking, as shown in Fig. 3.

\subsection{Construction of the Vehicle Model in the Dynamic Model}

To obtain the lateral velocity, slip rate, roll angle, yaw velocity and sideslip angle, the vehicle dynamic model was 
built. As a complex rigid body, the vehicle is reasonably simplified by the dynamic model to become more accessible to the actual cornering braking condition. In Fig. 3 , this model is a combination of the driving model, tire model, vehicle model and auxiliary calculation model. The tire model uses the most classic model, which is the GIM model. An eight-degree-of-freedom vehicle model is a common analysis model. In the cornering braking process, because turning control should be added to the system based on the four running wheels, four wheels of an eight-degree-of-freedom vehicle model were built. In figure 4, the eight degrees of freedom of the model are the longitudinal motion, lateral motion, yawing motion, rolling motion of the vehicle body and the running of four wheels.

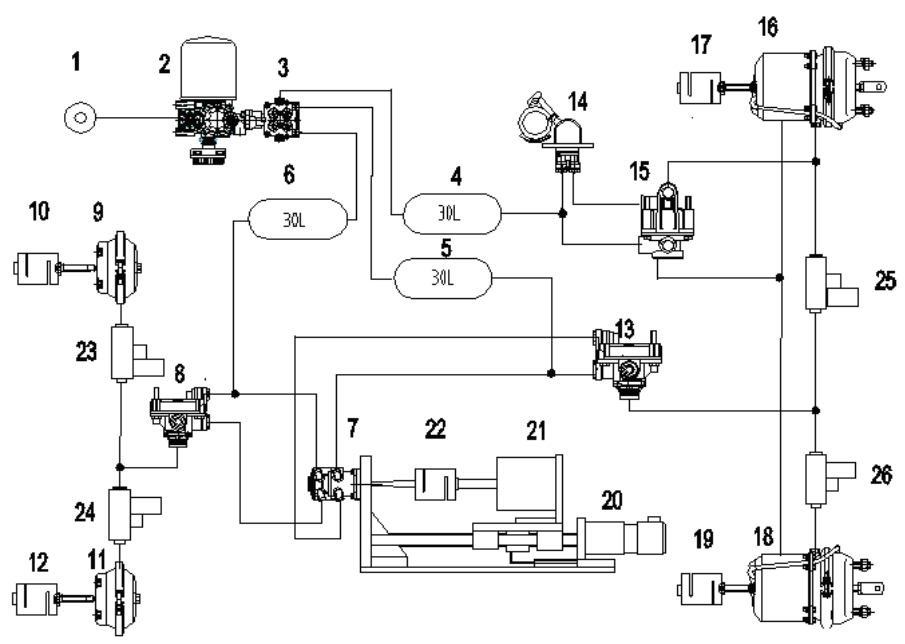

Figure 2 Hardware components of the air brake system:

1 - Air source; 2 - Air dryer; 3 - Four-circuit valve; 4, 5, 6 - Air reservoir; 7 - Total brake valve; 8 - Front axle relay valve; 9, 11- Diaphragm brake chamber; 13 - Rear axle relay valve; 14 - Hand brake valve; 15 - Differential Relay Valve; 16, 18 - Rear axle spring brake cylinder; 20 - Servo motor; 21 - Quick cylinder; 10, 12, 17, 19, 22 - Force sensor; $23,24,25,26$ - ABS regulator.

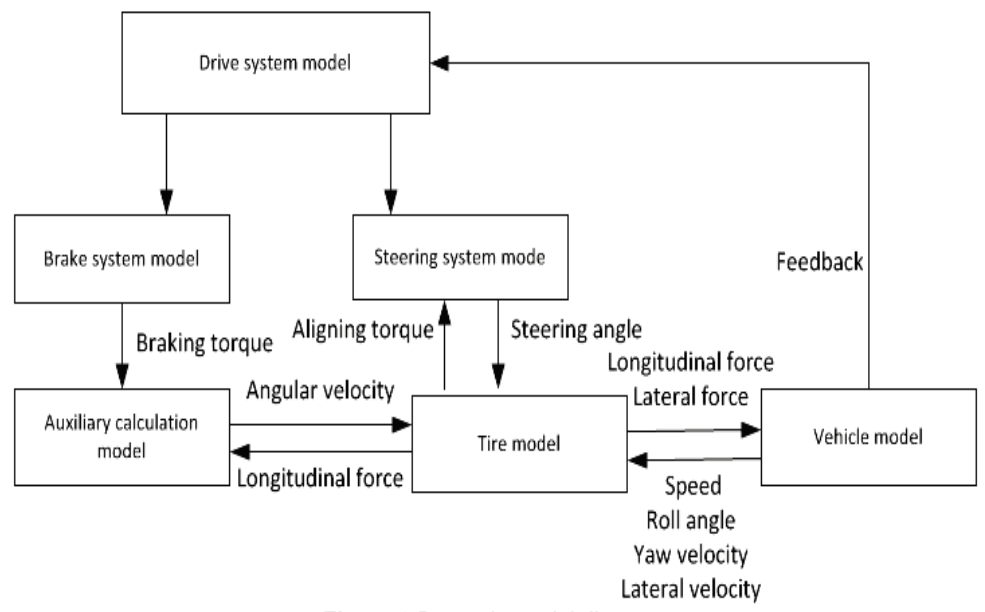

Figure 3 Dynamic model diagram

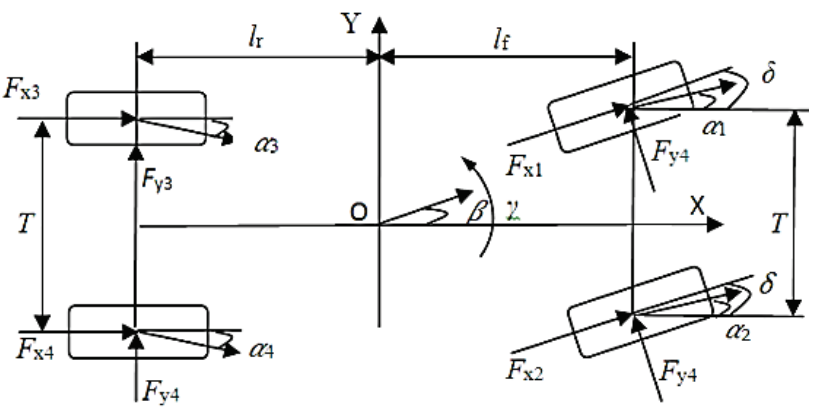

Figure 4 Four wheels of the eight-degree-of-freedom vehicle model

In the eight-degree-of-freedom vehicle model, the basic equation of vehicle running is:

$$
\begin{aligned}
& \left\{\begin{array}{l}
\sum F_{x}=M(\dot{u}-v \gamma) \\
\sum F_{y}=M(\dot{v}+u \gamma) \\
I_{z}=\sum M_{z} \\
I_{x} \dot{p}=\sum M_{x}+m_{s} h_{s}(\dot{v}+u \gamma) \\
I_{w} \frac{\mathrm{d} w}{\mathrm{~d} t}=F_{x b}-T_{b}
\end{array}\right. \\
& \sum F_{x}=\left(F_{x 1}+F_{x 2}\right) \cos \delta-\left(F_{y 1}+F_{y 2}\right) \sin \delta+F_{x 3}+F_{x 4}(2) \\
& \sum F_{y}=\left(F_{y 1}+F_{y 2}\right) \cos \delta+\left(F_{x 1}+F_{x 2}\right) \sin \delta+F_{x 3}+F_{x 4}(3)
\end{aligned}
$$


$\sum M_{z}=\left(-F_{x 1} \cos \delta+F_{y 1} \sin \delta-F_{x 3}+F_{x 2} \cos \delta-\right.$

$\left.-F_{y 2} \sin \delta+F_{x 4}\right) \cdot \frac{T}{2}+\left(F_{x 1} \sin \delta+F_{y 2} \cos \delta+\right.$

$\left.+F_{x 2} \sin \delta-F_{y 1} \cos \delta\right) \cdot l_{f}-\left(F_{y 3}-F_{y 4}\right) \cdot l_{r}$

Where $F_{x}$ is the tire longitudinal force $(\mathrm{N})$ (the left front wheel is $F_{x 1}$; the right front wheel is $F_{x 2}$; the left rear wheel is $F_{x 3}$; the right rear wheel is $\left.F_{x 4}\right) ; F_{y}$ is the tire lateral force (N) (the left front wheel is $F_{y l}$; the right front wheel is $F_{y 2}$; the left rear wheel is $F_{y 3}$; the right rear wheel is $\left.F_{y 4}\right) ; M$ is the body weight $(\mathrm{kg}) ; u$ is the speed $(\mathrm{km} / \mathrm{h}) ; v$ is the lateral velocity $(\mathrm{km} / \mathrm{h}) ; M_{z}$ is the body around the $\mathrm{z}$-axis of the coordinate system $(\mathrm{N} \cdot \mathrm{m})$; yawing moment $I_{x}$ is the suspension quality around the rotational inertia of the $\mathrm{x}$-axis $\left(\mathrm{kg} / \mathrm{m}^{2}\right) ; M_{s}$ is the vehicle suspension mass $(\mathrm{kg})$; $h_{s}$ is center of mass to the roll center distance (m); $g$ is the acceleration of gravity $\left(\mathrm{m} / \mathrm{s}^{2}\right) ; \gamma$ is the yawing angular velocity $(\mathrm{rad} / \mathrm{s}) ; I_{z}$ is the rotational inertia of the body around the z-axis of the coordinate system $\left(\mathrm{kg} / \mathrm{m}^{2}\right) ; I_{w}$ is the moment of inertia of the wheel $\left(\mathrm{kg} / \mathrm{m}^{2}\right) ; w$ is the wheel rotating angular velocity $(\mathrm{rad} / \mathrm{s}) ; F_{x b}$ is the ground braking force $(\mathrm{N}) ; T_{b}$ is the breaking force that acts on the wheel $(\mathrm{N}) ; \delta$ is the wheel angle (rad), $p$ is the speed of roll angle $(\mathrm{rad} / \mathrm{s}) ; \dot{p}$ is the roll angle acceleration $\left(\mathrm{rad} / \mathrm{s}^{2}\right) ; \varphi$ is the roll angle (rad); $l_{f}$ is the center of mass to the front axle distance $(\mathrm{m}) ; l_{r}$ is the center of mass to the rear axle distance $(\mathrm{m}) ; k_{i}(i=f, r)$ is the roll angle stiffness of the front axle and rear axle $(\mathrm{N} \cdot \mathrm{m} / \mathrm{rad}) ; C_{\mathrm{i}}(i=f, r)$ is the roll angle damping of the front axle and rear axle $(\mathrm{Ns} / \mathrm{rad}) ; T$ is the wheel tread $(\mathrm{m})$. The concrete numerical parameters are shown in Tab. 1

Table 1 Vehicle parameter
\begin{tabular}{|l|c|c|}
\hline \multicolumn{1}{|c|}{ Parameter } & Symbolic unit & Value \\
\hline Total weight & $M(\mathrm{~kg})$ & 10500 \\
\hline Height of center of mass & $h(\mathrm{~m})$ & 1.2 \\
\hline Moment of inertia of the $\mathrm{z}$ axis & $I_{\mathrm{z}}\left(\mathrm{kg} / \mathrm{m}^{2}\right)$ & 50537 \\
\hline The wheel vertical stiffness & $C_{\mathrm{s}}(\mathrm{kN} / \mathrm{rad})$ & 200 \\
\hline The wheel lateral stiffness & $C_{\mathrm{a}}(\mathrm{kN} / \mathrm{rad})$ & 26 \\
\hline The wind resistance coefficient & $C_{\mathrm{d}}$ & 0.6 \\
\hline Moment of inertia of the wheel & $J\left(\mathrm{~kg} / \mathrm{m}^{2}\right)$ & 25 \\
\hline The wheel rolling radius & $R(\mathrm{~m})$ & 0.5715 \\
\hline $\begin{array}{l}\text { The center of mass to the front } \\
\text { axle distance }\end{array}$ & $a(\mathrm{~m})$ & 2.742 \\
\hline $\begin{array}{l}\text { The center of mass to the rear } \\
\text { axle distance }\end{array}$ & $b(\mathrm{~m})$ & 1.258 \\
\hline Wheel base & $T(\mathrm{~m})$ & 1.91 \\
\hline Braking efficiency factor & $\kappa$ & 3.2 \\
\hline efficiency coefficient & $\eta$ & 0.92 \\
\hline Windward area & $A\left(\mathrm{~m}^{2}\right)$ & $1.9 \times 2.8$ \\
\hline
\end{tabular}

Using formulas (1), (4) and (5) with the initial velocity, angle of the vehicle, braking torque and vehicle parameters, we can obtain the yawing angular velocity, centroid side-slip angle, roll angle, lateral acceleration, etc. Using formulas (3) and (4) with the side angle and tire force, we obtain the vehicle longitudinal and lateral force.

\subsection{Analysis on the Stability of Cornering Braking 2.3.1 Stability Control of Cornering Braking}

The speed, yaw velocity and sideslip angle, which reflect the running stability of the vehicle, are commonly nonlinear when the vehicle is attached to a road surface or a corner at high speed or brakes with large steering [8]. It is difficult for the driver to directly perceive the three variables and make the correct adjustment, which results in traffic accidents. Generally, the slip rate of 0.2 is used as the control target when the vehicle is in linear motion. However, during cornering braking, it is difficult to improve the stability of the vehicle simply by controlling the slip ratio. Therefore, this article suggests taking the sideslip angle and yaw velocity as the stability control parameters; decisions are made by comparing the differences between the target value and the ideal value, and feedback is given to the slip rate, which changes the yawing moment to improve the running stability of the vehicle.

The yaw velocity and sideslip angle are closely related to the vehicle stability. When the sideslip angle is small, the yaw velocity can reflect the cornering ability of the vehicle. With the increase in yaw velocity, the turn radius decreases and becomes more intense, which fully embodies the essential properties of the cornering movement. In this sense, the two variables must be controlled in the permitted range (expected value) [9].

To obtain these two parameters, this article directly takes the front wheel as the subject; the effect of the steering system and suspension are ignored. The vehicle body only moves in parallel to the ground. Thus, a simple mathematical model with only the side angle and yaw is established to reflect the basic characteristics of the curvilinear movement of the vehicle, and the ideal yaw velocity and sideslip angle can be acquired using the simultaneous equation. See Fig. 5.

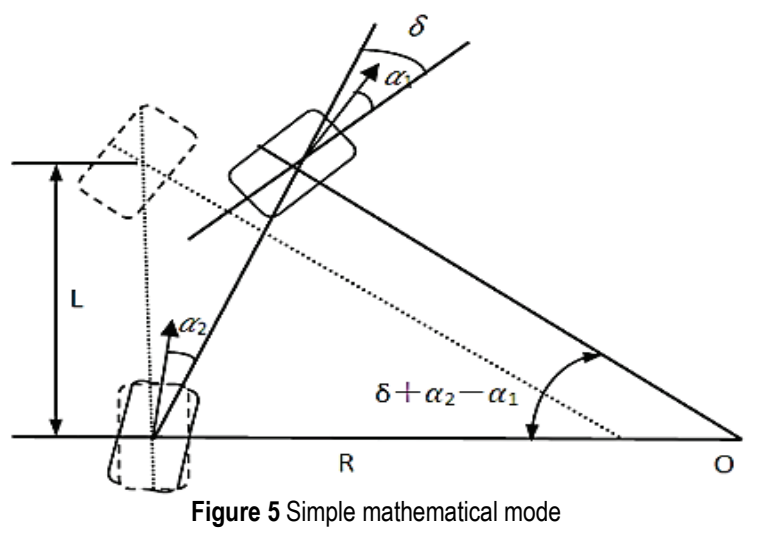

In this figure, $\delta$ is the steering angle ( $\mathrm{rad}), \alpha_{1}$ is the front-wheel sideslip angle (rad), $\alpha_{2}$ is the rear-wheel slip angle (rad), $R$ is the turning radius ( $\mathrm{m})$, and $L$ is the axle distance $(\mathrm{m})$. The turning radius is controlled by the side-slip angle. Fig. 5 shows the sine theorem:

$$
R \approx \frac{L}{\delta+\alpha_{2}-\alpha_{1}}
$$

The component of the centroid acceleration in the $y$ direction is:

$$
a_{y}=\dot{v}+u \gamma
$$


$\dot{v}$ is the lateral acceleration $\left(\mathrm{m} / \mathrm{s}^{2}\right)$.

The yaw velocity is:

$$
\begin{aligned}
& \gamma=\frac{v}{R}=\frac{v}{L}\left(\delta+m a_{y} \frac{l_{f}}{k_{2} L}-m a_{y} \frac{l_{r}}{k_{1} L}\right)= \\
& =\frac{\frac{\delta u}{L}}{1+\frac{m}{L^{2}}\left(\frac{l_{f}}{k_{2}}-\frac{l_{r}}{k_{1}}\right) u^{2}}=\frac{\frac{\delta u}{L}}{1+K u^{2}}
\end{aligned}
$$

Where:

$$
K=\frac{m}{L^{2}}\left(\frac{l_{f}}{k_{2}}-\frac{l_{r}}{k_{1}}\right)
$$

$k_{1}$ and $k_{2}$ denote the total cornering stiffness $(\mathrm{N} / \mathrm{rad})$ of the front and rear wheels, respectively, $K$ is the stability factor, and the steady-state response of the vehicle can be determined by $K$, if $\alpha_{1}-\alpha_{2} \geq 0, K>0$, the vehicle has the characteristics of under steer or neutral steer; if $\alpha_{1}-\alpha_{2}<0$, $K<0$, the vehicle has the over-steer characteristics.

In the limit state and with the limit of the wheel adhesion coefficient, the maximum expected yaw velocity is:

$$
\gamma=\frac{\mu \cdot g}{v}
$$

where $\mu$ is the road adhesion coefficient.

Based on the above analysis, the revised expected yaw velocity is:

$\gamma_{d}=\min \left\{\left|\frac{v}{\left(1+K v^{2}\right) L} \delta\right|,\left|\frac{\mu \cdot g}{v}\right|\right\} \cdot \operatorname{sign}(\delta)$

In $\operatorname{sign}(x)$, when $x>0, \operatorname{sign}(x)=1$; when $x=0, \operatorname{sign}(x)$ $=0$; when $x<0, \operatorname{sign}(x)=-1$.

When the vehicle is attached to the road surface, the simple yaw velocity control cannot achieve the desired effect, and the sideslip angle is another control variable. Since the lateral force of the wheel is saturated to a certain extent and causes the vehicle to lose the lateral stability, it is important to limit the sideslip angle and ensure that the wheel lateral force does not reach the boundary saturation to avoid dangerous conditions. The boundary saturation is expressed as follows [10]:

$\left|B_{1} \dot{\beta}+\beta\right|=\mu B_{2}$

$\beta$ is the sideslip angle (rad); $\dot{\beta}$ is the differential of the sideslip angle; $B_{1}$ and $B_{2}$ are the correlation coefficients. Through simulation analysis, $B_{1}=0.32$, and $B_{2}=0.10$, so the saturation region is:

$\left|B_{1} \dot{\beta}+\beta\right| \leq \mu B_{2}$
Based on the above analysis, the deviations between the actual yaw velocity and sideslip angle and the expected values are calculated using formulas (14) and (15):

$$
E_{\gamma}=\gamma_{d}-\gamma_{0}
$$

$E_{\beta}=\left\{\begin{array}{cc}0 & \left|B_{1} \dot{\beta}+\beta\right|<\mu B_{2} \\ \frac{\left|B_{1} \dot{\beta}+\beta-\mu B_{2}\right|}{\sqrt{1+B_{1}^{2}}} & \left|B_{1} \dot{\beta}+R \beta\right| \geq \mu B_{2}\end{array}\right.$

$\gamma_{0}$ is the actual yaw velocity $(\mathrm{rad} / \mathrm{s})$.

\subsubsection{A Control Module of the Threshold Value of the Slip Rate based on the Fuzzy-PID Control Algorithm}

Because of the nonlinearity and time-varying problems in controlling the yaw velocity and sideslip angle, a fuzzy PID control algorithm is used to check and adjust the two controlled variables, so that the actual value matches the desired value. Fig. 6 shows the block diagram of the PID control algorithm of the threshold value adjustment of the slip rate. We establish the fuzzy controllers of the yaw velocity and sideslip angle, calculate their threshold values with the fuzzy PID control algorithm, and synthesize these data to obtain the ideal slip rate [11]. The fuzzy domain of the input and output is defined as $[-1,1]$; thus, there are five linguistic variables in the domain: Negative Big (NB), Negative Small (SB), zero (Z), Positive Small (PS) and Positive Big (PB).

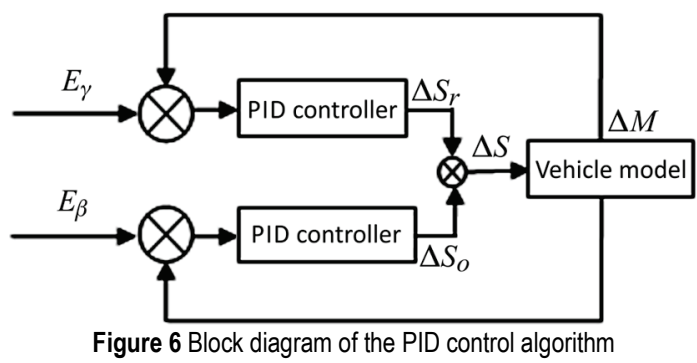

\subsection{RESULTS AND DISCUSSION}

Under the condition of the step angle input for the front wheel, the vehicle began to turn at the $4^{\text {th }}$ second and reached the maximum angle of approximately $0.04 \mathrm{rad}$ at the 5th second. Fig. 7 shows the cornering braking of the vehicle with the initial speed of $60 \mathrm{~km} / \mathrm{h}$ on a road surface with high adhesion coefficient (the rolling adhesion coefficient is 0.84 , and the slip adhesion coefficient is $0.75)$. The vehicle began to brake after 2.2 seconds and ended after 10.7 seconds with uniform speed changes. The slip rates of the left and right front wheels were controlled at approximately 0.6 , and those of the left and right rear wheels were controlled at 0.2 , which slightly decreased in the end. The lateral speed began to increase to $8 \mathrm{~km} / \mathrm{h}$ and finally decreased to nearly 0 when the vehicle slowed, which indicates that the vehicle did not drift or side slip. The yaw velocity reached the peak of $0.5 \mathrm{rad} / \mathrm{s}$ after turning, which indicates that the yaw velocity was under control. The sideslip angle was also maintained at the stable corresponding value, and the final rapid increase 
was caused by the stop of the vehicle, when the ABS no longer worked. Since the yaw velocity and sideslip angle were under effective control, the increase of some braking force did not make the vehicle lose stability.
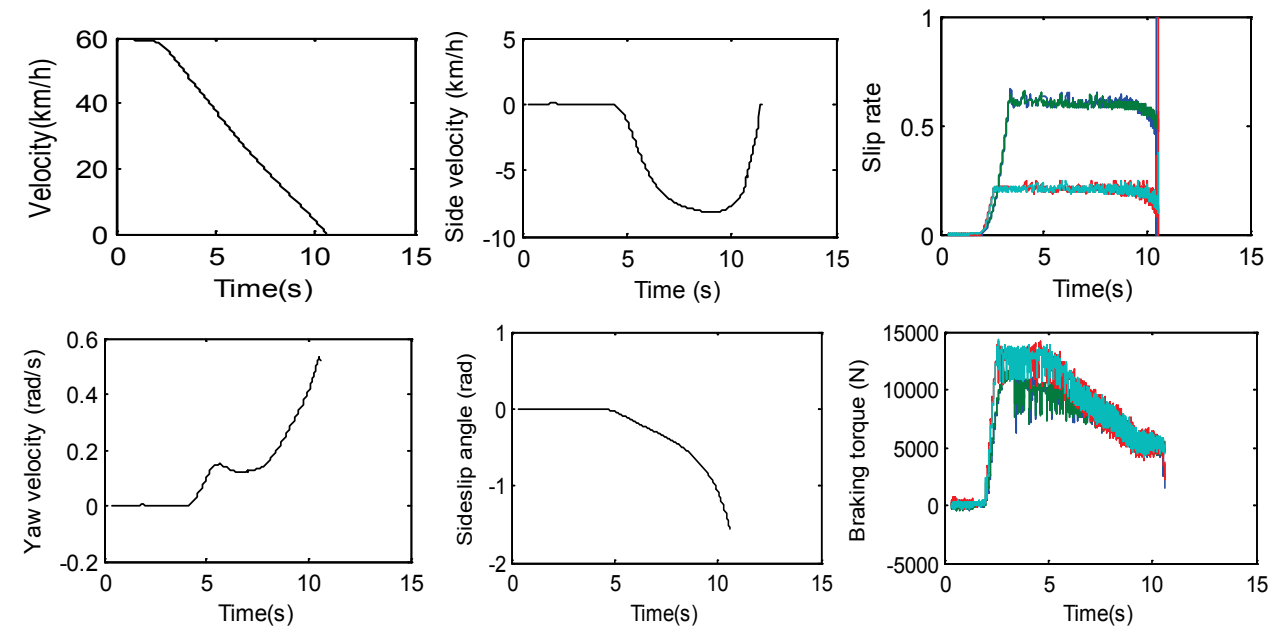

Figure 7 Results of the high-adhesion-coefficient pavement test
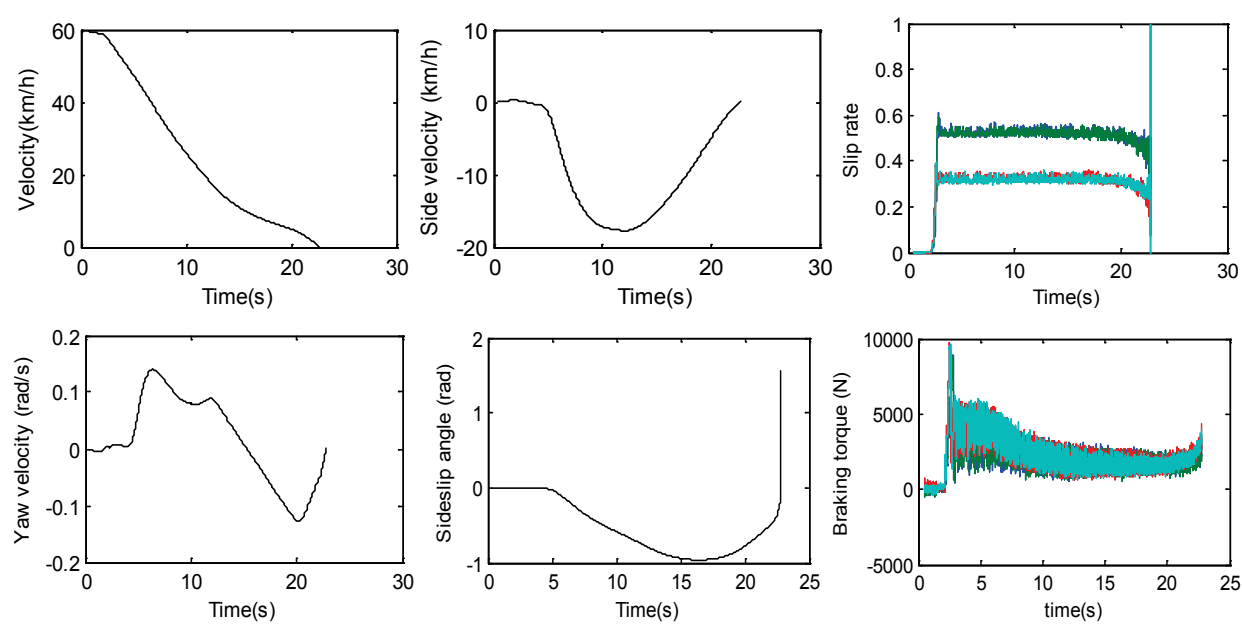

Figure 8 Results of the low-adhesion-coefficient pavement test

Fig. 8 shows the cornering braking of the vehicle with the initial speed of $60 \mathrm{~km} / \mathrm{h}$ on a road surface with low adhesion coefficient (the rolling adhesion coefficient is 0.3 , and the slip adhesion coefficient is 0.22 ). The vehicle began to brake after approximately 2.5 seconds and ended after 23 seconds, i.e., the process lasted approximately 20.5 seconds. Compared to the road surface with a high adhesion coefficient, the braking time was longer for the road surface with low adhesion coefficient because the ground provided a smaller force, and the braking distance also increased. The lateral speed eventually decreased to almost zero after the sharp increase at the beginning. The slip rate of the front wheel was controlled at approximately 0.5 , and that of the rear wheel was controlled at 0.3 . The vibration was notably fast in this condition, which shows that the wheel did not lock. The maximum yaw velocity was approximately $0.1 \mathrm{rad} / \mathrm{s}$, which is notably small. The braking moment frequently changed; the vehicle was stable and under control during the entire braking process.

\section{CONCLUSIONS}

The cornering stability of the vehicle was improved based on the traditional ABS control when the vehicle turned at high speed. By ignoring the theoretical value of
0.2 , the expected value and actual value of the yaw velocity and sideslip angle were compared using the fuzzy PID algorithm to calculate the ideal threshold slip rate to control the yawing moment, and accidents such as spin and side slip can be avoided. In the future, in order to further improve the braking stability of system, consider decomposition brake force, using the corresponding yawing moment, makes the control of $\mathrm{ABS}$ and yawing moment more coordination.

\section{Acknowledgments}

This work is supported by the State Quality Inspection Administration Public Welfare Scientific Research Project of China (No. 201310284) and Zhejiang Province Public Technology Research Program (No. 2015C31081).

\section{REFERENCES}

[1] Yang, J. P. (2008). On the Discrete Transient Aerodynamic Coefficient of Cars Crosswind Stability Study. Chin. Mech. Eng., 27(4), 549.

[2] Zhang, S., Sun, T. J. \& Kang, W. X. (2006). Hybrid Modeling and Simulation of Vehicles ABS Based on Simulink/Stateflow. Bus Coach Tech. Res., 23(02).

[3] Yu, Q., Chen, Y. S. \& Ma, J. (2003). Affect of Engine Brake 
and Exhaust Brake on Bus Brake Stability. J. Trf. Trans. Eng., 3(3), 64.

[4] Gawthrop, P. J., Virden, D. W. \& Neild, S. A. (2008). Emulator-based Control for Actuator-based Hardware-in-the-Loop Testing. Ctrl. Eng. Practice, 16(8), 897. https://doi.org/10.1016/j.conengprac.2007.10.009

[5] Yang, T. \& Song, D. D. (2012). Simulation Study of Vehicle Brake Stability Control on Turning Lane Based on ABS. Adv. Mater. Res., 591. https://doi.org/10.4028/www.scientific.net/AMR.591-593.1916

[6] Shen, F. P., Zhao, Y. Q. \& Zhao, H. G. (2015). The Nonlinear Tire Lateral Force Effect on the Vehicle Steering Stability. Chin. Mech. Eng., 26(1), 135.

[7] Yang, D., Li, L. T. \& Yang, X. (2002). The System Real-time Simulation Development Environment and Application. Tsinghua University Press, Beijing.

[8] Yu, F., Xu, Z. M. \& Zhang, Z. F. (2010). Fuzzy Control and Stability of Vehicle Cornering Brake ABS Simulation. $J$ Sci. Tech. $R \& D, 32(1), 782$.

[9] Chen, W. W., Lu, X. Y. \& Huang, H. (2012). Considering the Effects of Road Vehicle Stability Control Center of Mass Side-slip Angle Dynamic Boundary Control, J. Mech. Eng., 48(14), 112. https://doi.org/10.3901/JME.2012.14.112

[10] Wang, Z. Z., Yu, L. Y. \& Song, J. (2014). Based on the Braking System of Vehicle Wheel Slip Rate Control Research Status. J. Auto. Eng., 36(1), 81.

[11] Li, C., Li, X. H. \& Jin, J. (2014). Fuzzy Control of Tractor-semitrailer Cornering Braking Stability. J. $\mathrm{HW}$ Trans. $R \& D$., 31(11), 147.

\section{Contact information:}

Yi LU, Associate professor

School of Metrology \& Measurement Engineering,

China Jiliang University

No. 258, Xueyuan Street, Higher Education Zone of Xiasha,

310018 Hangzhou, Zhejiang, China

E-mail: luyi9798paper@163.com

Corresponding author: Yi LU

\section{Jie LU, Postgraduate}

School of Metrology \& Measurement Engineering,

China Jiliang University

No. 258, Xueyuan Street, Higher Education Zone of Xiasha,

310018 Hangzhou, Zhejiang, China

E-mail: ludim9404@163.com

\section{Jia-wei WU, Postgraduate}

School of Metrology \& Measurement Engineering,

China Jiliang University

No. 258, Xueyuan Street, Higher Education Zone of Xiasha,

310018 Hangzhou, Zhejiang, China

E-mail: 742847301@qq.com

\section{Bin GUO, Associate professor}

School of Metrology \& Measurement Engineering,

China Jiliang University

No. 258, Xueyuan Street, Higher Education Zone of Xiasha,

310018 Hangzhou, Zhejiang, China

E-mail: guobin905@163.com 